\title{
Ciclos y élites: patrones recurrentes en la literatura de Asimov
}

Recibido: 26/03/2018 | Revisado: 22/10/2018 | Aceptado: 13/11/2018 DOI: $10.17230 /$ co-herencia.16.30.8

\author{
José Luis Arroyo* \\ jlarroyo@comillas.edu / jlarroyob@us.es
}

Resumen La repetición de patrones es, junto a la riqueza transtextual de su obra, un elemento característico en la literatura de Isaac Asimov. Ambos configuran un estilo muy particular, criticado en ocasiones, pero indudablemente interesante, pues permite una lectura palimpséstica que los aficionados al género de la ciencia ficción siempre han valorado. En el caso concreto de los patrones, hay dos que se repiten con gran frecuencia en sus distintos trabajos: los ciclos y las élites gobernantes. Respecto al primero, Asimov usa una y otra vez la idea de ciclo histórico, insistiendo en una suerte de palingenesia, en ocasiones de un modo sutil, y en otras casi según la interpretación literal que hacían los estoicos, como sucede en el relato "Nightfall". Acerca del segundo, las élites gobernantes, inspiradas en gran medida en La República de Platón, observamos claras semejanzas intertextuales entre sus obras, con esquemas extraordinariamente similares. En este trabajo profundizamos en ambos patrones, recorriendo algunas de sus principales novelas y relatos para esclarecer los diseños que Asimov repetía con asiduidad.

\section{Palabras clave:}

Literatura comparada, tematología, ciencia ficción, transtextualidad, racionalidad.

\section{Cycles and elites: recurrent patterns in Asimov's works}

\footnotetext{
Abstract The repeating patterns, along with the transtextual richness, are characteristic elements in the writings of Isaac Asimov. Both make up a very particular style, criticized at times, but undoubtedly interesting, because they enable a palimpsest-like reading that fans of science fiction enthusiasts have always appreciated. Regarding patterns, there are two that are often repeated in his different works: the cycles and the governing elites. As for the first pattern, Asimov uses over and over again the idea of the historical cycle, insisting on a sort of palingenesis, sometimes in a subtle way, and others almost in line with the literal interpretation by the Stoics, as in the story "Nightfall". About the second pattern, the governing
}

Doctor por la Universidad Complutense de Madrid, España. Profesor en la Universidad Pontificia Comillas, España. Investigador en la Universidad de Sevilla, España. ORCID: 00000002-3660-3933. 
elites, inspired largely by Plato's Republic, clear intertextual similarities can be observed among his works, with extraordinarily similar schemes. In this paper we delve into both patterns, exploring some of his main novels and stories in order to shed light on the schemes assiduously repeated by Asimov.

\section{Keywords:}

Comparative literature, thematology, science fiction, transtextuality, rationality.

Una de las señas de identidad de Isaac Asimov, tanto en su faceta de autor de ciencia ficción, como en la de divulgador, era su enorme fe en la ciencia y la tecnología. ${ }^{1}$ En una de sus últimas entrevistas, llegó a afirmar que la

[...] technology can save us [...]. I don't know for sure... some of our problems may prove insoluble even for science and technology. But if those two tools fail us, nothing else will succeed (Asimov, en Stone, 2005 , p. 61). ${ }^{2}$

Su opinión sobre la ciencia, como una fuerza benevolente con la capacidad de mejorar sensiblemente las condiciones de vida de la humanidad (Booker, 2001, p. 90), estaba muy presente en toda su producción novelística (Stableford, 1995, p. 48; Weldes, 2001), y ha sido criticada, en ocasiones con dureza, por diversos académicos. Como señala Sutherland, "Asimov offers a technological-utilitarian philosophy founded on an articulate atheism" (2014, p. 177), ${ }^{3}$ pues no solo consideraba la tecnología como el mejor indicador para entender una sociedad (Berger, 1979), sino también como la mejor herramienta para superar sus dificultades (Fitting, 1979; Milner y Savage, 2008). Clute (2007) apunta que, para el lector moderno, uno de los principales problemas de la trilogía Foundation, obra icónica de Asimov, es, precisamente,

1 O en términos más generales, la racionalidad. Gunn (2005b, pp. 36-37) señala que esta enorme confianza de Asimov en la racionalidad probablemente era consecuencia de algunas limitaciones personales durante su adolescencia y juventud, así como de un mecanismo de defensa ante los convulsos tiempos que hubo de vivir, y que incluyeron la Gran Depresión estadounidense y la Segunda Guerra Mundial.

2 [[...] la tecnología puede salvarnos [...]. No estoy seguro.... algunos de nuestros problemas pueden resultar irresolubles incluso para la ciencia y la tecnología. Pero si esas dos herramientas nos fallan, ninguna otra cosa tendrá éxito].

3 [Asimov ofrece una filosofía tecnológico-utilitaria basada en un elocuente ateísmo]. 
el cientifismo ${ }^{4}$ que se halla en conceptos como la psicohistoria. ${ }^{5}$ No es de extrañar que, como consecuencia de todo ello, los personajes asimovianos se caractericen por su extraordinaria y, de hecho, casi inhumana racionalidad (Martínez, 2012, p. 195; Palumbo, 2016, p. 2), e incluso que la acción de sus obras siga, paso por paso, el método científico ${ }^{6}$ (Warrick, 1977, p. 179), con una estructura narrativa bastante próxima a la de la novela policiaca (Käkelä, 2016, p. 15).

Los patrones y ciclos, como constante en la obra de Asimov, no son sino un reflejo de este pensamiento racionalista, ${ }^{7}$ una imagen de su concepción científica del mundo. ${ }^{8}$ La ciencia es, en última instancia, la búsqueda de patrones cuantificables en el lenguaje de las matemáticas, y esta idea permeó igualmente en la producción novelística de Asimov, pues no podemos olvidar que, a pesar de ser un notable humanista, era también un científico, ${ }^{9}$ un profesor universitario de bioquímica, moldeado en su pensamiento según el esquema propio de la instrucción en ciencias, cuyo denominador común es la indagación en el caos aparente para encontrar estructuras ordenadas, que ayuden a clasificarlo, explicarlo y, en última instancia, domeñarlo. Su obra más emblemática, la metasaga ${ }^{10}$ Foundation, es un caleidoscopio de patrones que se repiten a distintos niveles, tanto del cronotopo ${ }^{11}$ como de las tramas e incluso de la propia estructura, pero los patrones son una constante en toda su obra. Como señala Chaves, desde el área tematológica de los estudios de literatura comparada, ya se ha señalado que

4 Tendencia a dar un valor excesivo a los conocimientos científicos.

5 Ficticia rama de las matemáticas que, en las novelas de Asimov, permite predecir la evolución histórica de una sociedad.

6 Recogida y análisis de datos, formulación de una hipótesis, realización de predicciones sobre la posible solución al problema y verificación de las mismas.

7 Muchos académicos consideran que la ciencia ficción moderna se desarrolló a partir de la novela gótica (Roberts, 2017, p. 87), siendo la principal diferencia entre ambas la relevancia que la razón y la ciencia tienen en la primera (Roberts, 2017, p. 89).

8 Goble (1972, p. 22), por mencionar un caso concreto, sostiene que Asimov sentía verdadera fascinación por los patrones, tanto en ciencia como en otras disciplinas.

9 Aunque con algunos matices, pues como apunta Martínez (2012, p. 72), si bien parecía especialmente dotado para la docencia, sus capacidades en el ámbito de la investigación eran bastante mediocres.

10 Nos referimos a un conjunto de sagas que, al estar relacionadas y compartir ciertos elementos, pueden interpretarse como partes de una obra mayor, la metasaga.

11 Bajtín define el cronotopo como "la conexión esencial de relaciones temporales y espaciales asimiladas artísticamente en la literatura" (1989, p. 237). 
[...] en la literatura, paulatinamente el mito se torna en "tema", desde un cierto enfoque narrativo y argumental específico, en un texto literario concreto, que se vuelve luego susceptible de nuevas lecturas, esto es, de ser reformulado por otros autores en textos diferentes, y que van conformando así tradiciones y cadenas temáticas textuales, alimentadas con los desplazamientos semánticos de dicho tema, con repeticiones innovadoras a partir de un cierto perfil reconocible (2016, p. 78).

Esto es, exactamente, lo que sucede con la producción novelística de Asimov, solo que acontece en los textos del propio autor. Bien es cierto que Asimov incluye en sus obras determinados motivos o "temas" de otros escritores, como, por ejemplo, el patrón cosmogónico de Donald A. Wollheim, que se analiza más adelante en el apartado "Ciclos", o el propio carácter cíclico de la historia, aunque en este trabajo nos centramos en la repetición de sus propios motivos.

A lo largo de este artículo analizamos dos de estos patrones que, desde nuestro punto de vista, presentan un especial interés. El primero es el ciclo destrucción-creación, muy relacionado con su concepción recurrente de la historia, y que aparece tanto en sus relatos como en sus novelas. Se trata de un patrón con el que juega una y otra vez, aplicándolo a la evolución de las sociedades, ${ }^{12}$ del tiempo e incluso del propio universo. Como ya se ha indicado, la ciclicidad histórica no es algo exclusivo de Asimov, sino una tendencia relativamente frecuente en muchos escritores de ciencia ficción de la época, que, quizá influidos por Spengler o Toynbee, incorporaron el concepto en sus novelas (Shippey, Stableford, Feeley y Langford, 2015; Wolfe, 1986, p. 52). Desde una perspectiva tematológica, Shippey (2016, p. 84) señala que Campbell, ${ }^{13}$ el editor de ciencia ficción más

12 De hecho, la concepción asimoviana de la ciencia ficción tenía mucho que ver con predecir cambios sociales. Su definición de este género literario, muy popular entre los aficionados, es la siguiente: "sf is the branch of literature which deals with the response of human beings to changes in the level of science and technology" [La ciencia ficción es la rama de la literatura que se ocupa de las respuestas humanas a los cambios efectuados al nivel de la ciencia y la tecnología] (en Ingersoll, 2005, p. 22). Bowler (2017, p. 2), en esta misma línea, señala que Asimov, al igual que otros escritores de la época, trataban de predecir cómo podría evolucionar la sociedad, estando especialmente interesados en especular sobre cómo las nuevas tecnologías podrían crear o desviar las tendencias sociales existentes.

13 John W. Campbell, en un intento por redefinir la ciencia ficción de la época, reunió a un grupo de jóvenes escritores, entre los que se encontraba Asimov, generando una corriente que podríamos denominar "ciencia ficción social" (Novell Monroy, 2008, pp. 38-39). 
relevante de la época, fue quien trasladó a varios de sus escritores reflexiones relacionadas con el modelo cíclico de la historia, entre ellos al propio Asimov, siendo esta la causa por la que se trata de un motivo recurrente en la literatura de ese periodo. ${ }^{14}$

El segundo patrón, quizá no tan frecuente en su producción novelística, pero sin duda también muy interesante, es el de las élites gobernantes. Con una concepción muy próxima a la propuesta por Platón en La República, ${ }^{15}$ Asimov nos describe unas élites que, amparadas en su superioridad intelectual, rigen el destino de la humanidad, sin dudar lo más mínimo en emplear cualquier ardid necesario para alcanzar sus fines, sea este ético o no. La presencia de estas élites en la obra de Asimov quizá es consecuencia de lo que Käkelä (2014) describe como "tension between the Enlightenment freedom and the increasingly overpowering idea that society needs a mechanism to keep it on the right course - something that surpasses democracy, autocracy, or any 'regular' forms of governing". ${ }^{16}$ O puede que simplemente sea el reflejo literario de la idea de la salvación a través de las élites, compartida tanto por Estados Unidos como por la Unión Soviética en la época en que Asimov escribió sus principales novelas (Webster, 2015, p. 19). Sea cual fuere la causa, lo cierto es que se trata de un patrón que se repite en sus obras más emblemáticas, y que, por tanto, merece ser analizado en detalle.

14 Otros académicos matizan este particular. Pez (s. f.), por ejemplo, señala que fue un amigo de Asimov quien en 1944 le prestó el primer volumen de A Study of History de Toynbee, lo que, en su opinión, influyó en la historia de Foundation que escribía en aquel momento, esto es, la primera parte de Foundation and Empire.

15 Así como a los planteamientos de Maquiavelo en El príncipe. En este sentido, Fanzo señala que, en la trilogía Fundación, Asimov "emphasizes the Machiavelian, behind the scenes political maneuverings which influence this history and prompt action rather than describing the action itself" (1965, p. 68) [enfatiza lo maquiavélico, mediante escenas políticas que ocurren entre bastidores, escenas que influyen en la historia y provocan la acción, en lugar de describir la acción en sí misma].

16 [tensión entre la libertad de la Ilustración y la idea, cada vez más poderosa, de que la sociedad necesita un mecanismo para mantenerla en el camino correcto, algo que supera a la democracia, la autocracia o cualquier forma "regular" de gobierno]. 


\section{Ciclos}

Comenzamos nuestra reflexión con The End of Eternity (Asimov, 2000), una novela que, dada su originalidad y riqueza transtextual, ${ }^{17}$ no ha sido, en nuestra opinión, suficientemente reconocida..$^{18} \mathrm{El}$ éxito de Foundation probablemente la eclipsó, al igual que sucedió con el resto de la producción novelística de Asimov. La trama, original para la época, sigue siéndolo al día de hoy, y arranca con una organización, La Eternidad, que, aprovechando su conocimiento sobre el viaje en el tiempo, altera la realidad mediante sutiles cambios, siempre bajo el idealista objetivo de mejorar la vida de la especie humana. Cuando un determinado siglo, por ejemplificar dicho objetivo, se ve azotado por un incremento sustancial del abuso de drogas, los abnegados miembros de La Eternidad realizan un pequeño cambio en el pasado, que conduce a una nueva realidad en que dicho problema ha sido erradicado por completo. Así pues, los eternos, permanentes custodios de la humanidad, rigen su destino en secreto, ocultando con el máximo cuidado la naturaleza de sus actividades.

Otra característica que convierte The End of Eternity en una obra notable es, como ya hemos dicho, su riqueza transtextual, quizá la mayor en toda la producción novelística de Asimov. Sin embargo, su lectura palimpséstica no resulta sencilla en absoluto, pues, a diferencia de lo que sucede en la metasaga Foundation, en donde las alusiones hipertextuales e intertextuales son de tipo humanístico, ${ }^{19}$ aquí nos enfrentamos a referencias puramente científicas, difíciles de percibir para muchos lectores. No es el objetivo de este trabajo profundizar en ello, pero mencionamos dos ejemplos que ilustran a la perfección lo que aquí apuntamos.

El primer ejemplo está relacionado con el modo en que se expone el descubrimiento, puramente empírico, del campo temporal, cuando aún se desconocían las bases científicas capaces de esclarecer su funcionamiento. Asimov nos narra la sorpresa de una comunidad

17 Seguimos aquí a Genette, que define la transtextualidad como "todo lo que pone al texto en relación, manifiesta o secreta, con otros textos" (1989, pp. 9-10).

18 Esta novela fue finalista a los Premios Hugo en 1956, que, sin embargo, ganó la obra Double Star, de Robert Heinlein.

19 Las principales referencias son a los trabajos de Gibbon, Spengler, Toynbee y Polibio, como ya se ha analizado, por ejemplo, en Arroyo Barrigüete (2018). 
científica fascinada por un ingenio que funciona, y que, sin embargo, no es capaz de explicar. Para mayor desconcierto, cuando años después se comprende la ciencia subyacente, el ingenio original no puede ser mejorado. Tal vez estamos ante una referencia de tipo intertextual al desarrollo de la termodinámica, pues si bien en 1816 Robert Stirling patentó su motor basado en un ciclo termodinámico de aire u otro gas, no fue hasta 1824 cuando Nicolas Léonard Sadi Carnot explicó los fundamentos teóricos de su invención. Adicionalmente, el motor Stirling es el único que presenta un rendimiento teórico que alcanza el máximo posible, determinado por el rendimiento del motor ideal de Carnot.

El segundo ejemplo está relacionado con la teoría del multiverso, una curiosa interpretación de la mecánica cuántica propuesta por el físico Hugh Everett en los años cincuenta del siglo Xx. En términos generales, su hipótesis es que existen infinitas realidades alternativas, determinadas por la incertidumbre cuántica, ${ }^{20}$ y este es el planteamiento que nos hace Asimov hacia el final de su novela, cuando el protagonista es informado de que su concepción de la realidad, única y determinada exclusivamente por el flujo temporal, es por completo errónea, pues existen muchas realidades diferentes que coexisten en el tiempo. Sin embargo, no podemos saber hasta qué punto Asimov se inspiró en las tesis de Everett, pues la novela se escribió en las mismas fechas en que los círculos académicos empezaban a hacerse eco de la hipótesis del multiverso. ${ }^{21}$

En esta novela se plantea un bucle de tiempo cerrado, de modo que son los propios eternos del futuro quienes, enviando al pasado a un miembro de la organización, provocan que La Eternidad sea creada, al dotar a la humanidad de los conocimientos necesarios para su formación. La pregunta que queda en el aire es quién descubrió, en primera instancia, la ciencia del viaje en el tiempo. Este tipo de paradoja es un recurso relativamente habitual en ciencia ficción, y en

20 Su planteamiento, en términos más técnicos, es que cada vez que la función de onda asociada a una partícula cuántica colapsa (se fija su estado), el universo se escinde en varios, adoptando en cada uno de ellos un estado diferente, cubriéndose todos los valores posibles.

21 Si bien su tesis doctoral se defendió en 1957, dos años más tarde de la publicación de The End of Eternity las teorías de Everett ya eran previamente conocidas en el ámbito académico, pues comenzó a trabajar en esta idea en 1954. 
particular en aquella que trata sobre los viajes en el tiempo, siendo "All You Zombies", de Robert A. Heinlein (1959), uno de los casos más conocidos.

Pero lo interesante en la obra de Asimov es el modo en que explora, por boca de los diferentes actantes, las posibles explicaciones y consecuencias. Los esfuerzos de los eternos para mantener el ciclo son el eje vertebrador de la novela. Por medio de un intricado plan, pretenden reproducir en el pasado exactamente los mismos acontecimientos que dieron lugar a la creación de su organización. Se trata, por tanto, de una novela que explora las paradojas del viaje temporal, pero con el foco puesto en el concepto de ciclo temporal cerrado, algo de hecho muy coherente con las ideas asimovianas de patrones que se repiten una y otra vez.

Si en la novela anterior Asimov exploraba los ciclos en el viaje temporal, en otras trata una idea de la que al parecer estaba convencido: $:^{22} \mathrm{el}$ carácter cíclico de la propia historia. Se suele mencionar a Spengler ${ }^{23}$ o Toynbee ${ }^{24}$ como fuente de inspiración en este sentido, con influencias también de historiadores griegos como Polibio. Sean cuales fueren sus fuentes de inspiración, lo cierto es que Asimov parecía concebir la historia como algo cíclico, conformado por patrones que se repiten una y otra vez. Así pues, en la metasaga Foundation, compuesta por un total de quince novelas, ${ }^{25}$ se desarrollan todas y cada una de las etapas del patrón cosmogónico de Wollheim, ${ }^{26}$ propio de

22 Patrouch (1974, p. 85) afirma que Asimov estaba convencido del carácter cíclico de la historia, lo que su producción novelística revela con claridad.

23 Véanse, entre otros, Correia Félix (2014, p. 18), Csicsery-Ronay Jr. (2008, p. 221) y Fitting (1979).

24 Véanse, entre otros, Correia Félix (2014, pp. 109, 112), Hassler (1988), Irwin (1997), Sawyer (2009, p. 491), Seed (2011, p. 100), Shippey (2016, p. 84) y White (2005, p. 85).

25 Siguiendo con los patrones, Palumbo $(1995 ; 2016)$ afirma que la metasaga sigue una estructura autosemejante de tipo fractal, con subsagas organizadas en tres grupos de libros compuestos, a su vez, de otros grupos de novelas.

26 Según señala Gunn, "In their concerns about the future, science-fiction writers have developed a consensus scenario. With remarkable agreement, particularly in the early years of Gernstback an then Campbell, science fiction writers evolved what Donald Wollheim called 'the full cosmogony of science-fiction future history"' [En su preocupación por el futuro, los escritores de ciencia ficción han desarrollado un escenario de consenso. Con un elevado grado de acuerdo entre ellos, especialmente en los primeros años de Gernstback y Campbell, los escritores de ciencia ficción desarrollaron lo que Donald Wollheim llamó "la cosmogonía completa de la historia futura de la ciencia ficción”] (2018, p. 212). 
los escritores de ciencia ficción de la época: ${ }^{27}$ exploración espacial, colonización de planetas, surgimiento y auge de un imperio galáctico compuesto por muchos mundos, caída del imperio, interregno caracterizado por la barbarie, vuelta a la civilización y surgimiento de un nuevo imperio. ${ }^{28}$

El primer conjunto de libros, compuesto por cinco novelas, conforman lo que se ha denominado el ciclo de los robots o ciclo de la Tierra. En ellos se exploran los primeros intentos de la humanidad por desarrollar robots y las consecuencias de la integración de estos en la sociedad (Asimov, 1950a), su utilización e influencia en el proceso de colonización espacial (Asimov, 1954; 1957; 1983) y el modo en que son los robots mismos quienes dan el impulso definitivo, propiciando que la raza humana inicie una expansión por toda la galaxia (Asimov, 1985). Estas obras cubren, por tanto, las dos primeras etapas, en las que se comienza, aún muy tímidamente, la exploración y la colonización de nuevos planetas.

El segundo grupo de libros, conocido como ciclo del Imperio, nos sitúa muchos años en el futuro, con más de mil mundos poblados (Asimov, 1951), para saltar posteriormente al momento en el que la Republica de Trantor comienza su expansión (Asimov, 1952), gestándose así el embrión de lo que llegará a convertirse en el Primer Imperio Galáctico.

El último libro de este grupo nos lleva aún más adelante, con el Imperio ya creado (Asimov, 1950b), y de este modo se completa la tercera etapa del patrón cosmogónico.

Para terminar, el ciclo de la Fundación queda conformado por siete obras, que comprenden tanto la trilogía nuclear de la saga, publicada entre 1951 y 1953 (Asimov, 2004b, 2004d, 2004g), como dos secuelas (Asimov, 2004c, 2004e) y dos precuelas (Asimov 2004a, 2004f), que cubren las últimas tres etapas. Este ciclo se inicia con la caída del Imperio Galáctico y los esfuerzos de un científico, Hari Seldon, por disminuir el periodo de barbarie que sobrevendrá a la

27 Véase James (2015, pp. 24-25).

28 Desde una perspectiva tematológica y con vistas a futuras investigaciones, sería interesante analizar cómo este motivo fue tratado por otros autores del periodo, pues, como se ha indicado, se trata de un patrón bastante común entre los escritores de ciencia ficción de la época. 
misma. Mediante la creación de dos fundaciones, custodias de la ciencia y el conocimiento, pretende reducir el interregno de barbarie a mil años, momento en que surgirá un Segundo Imperio, que devolverá el orden a la galaxia.

Como hemos comprobado, en la metasaga Foundation se nos presenta un ciclo de auge y decadencia, coherente con esta concepción repetitiva de la historia que mencionábamos. Pero esta idea de ciclos recurrentes que observamos a nivel global también se da a nivel de las tramas, conformando un mosaico de estructuras que se repiten a distinta escala.

La primera novela de la metasaga, Foundation, es un compendio de relatos con una estructura idéntica: en todos ellos, la recién creada Fundación se enfrenta a un desafío que amenaza con destruirla, y que tras solventar satisfactoriamente, hace que aumente su influencia en los planetas vecinos. Se trataría de un ciclo continuo de crisisexpansión.

Si alejamos el zoom hasta la trilogía nuclear, en estas tres novelas vemos la alternancia cíclica de las formas de gobierno que describe Polibio. ${ }^{29}$ Constituiría un ciclo cerrado de los distintos modelos de Estado.

Y, finalmente, cuando tomamos la suficiente perspectiva para evaluar la metasaga por completo, nos encontramos ya con el patrón cosmogónico descrito anteriormente. Sería un ciclo de auge y decadencia que comprende millones de años y toda la galaxia.

La misma idea queda reflejada en "Nightfall" (1941), ${ }^{30}$ uno de sus relatos más celebrados, que abunda en el concepto de los ciclos históricos, aunque con un enfoque aún más radical. En esta obra se describe una civilización extraterrestre, pobladores de un extraño mundo,

29 La anaciclosis, descrita por el historiador griego Polibio en su obra Historias, plantea un ciclo en las formas de gobierno, que se suceden en un orden determinado: "a cada una de las constituciones le es congénita y le acompaña por naturaleza una forma degenerada: a la realeza la forma llamada monárquica, a la aristocracia, la oligarquía y a la democracia, la forma animalesca y basada en la fuerza bruta [oclocracia]" (Polibio, 2008, p. 149). Polibio también afirma: "He aquí el ciclo completo de las constituciones, he aquí su orden natural de acuerdo con el cual las constituciones cambian y se transforman y nuevamente retornan a su estadio primitivo. Cuando alguien conoce en profundidad este tema [...] raramente se equivocará en cuanto al grado de desarrollo de cada uno o de decadencia o en qué se transformará [...]" (Polibio, 2008, p. 148).

30 Años más tarde convertiría este relato en una novela, coescrita con Robert Silverberg (Asimov y Silverberg, 1990). 
con seis soles, llamado Kalgash, en el que nunca hay oscuridad. Sin embargo, cada 2049 años, debido a la rotación de sus soles, se produce una noche sin luz, lo que lleva a todos sus habitantes a enloquecer y destruir por completo la civilización, que tras el amanecer debe ser reconstruida de nuevo, prácticamente desde cero. Estaríamos ante un ciclo de creación y destrucción, un eterno retorno similar a la palingenesia de los estoicos.

Pero Asimov lleva este eterno retorno aún más lejos cuando extiende el ciclo al universo en su conjunto en The Gods Themselves (1990). En esta novela nos ofrece una obra que, en su propia estructura, es una continua repetición de patrones. Como señala Hark (1979), la obra se compone de tres partes, en cada una de ellas hay tres personajes principales y la solución final pasa por el bombeo de electrones entre tres universos paralelos. Los nombres de los protagonistas de la segunda parte, Odeen, Dua y Tritt, fonéticamente muy similares a one, two, three, son otra pista más sobre esta estructura autosemejante.

Sin embargo, en nuestra opinión, lo más interesante es que, en medio de esta repetición de patrones, la novela termina cuando un científico proporciona una explicación al big bang y, por tanto, al origen de nuestro universo. En su búsqueda de soluciones al problema que amenaza con destruir el sistema solar, esto es, el debilitamiento de la fuerza nuclear fuerte, inicia el bombeo de energía con otro universo embrionario, en el que al alterar sus leyes físicas provoca un big bang artificial y posibilita de ese modo la aparición de vida: "Indeed, it is by inducing the conditions required for the big bang that we may be setting up a new land of Universe that will eventually grow hospitable to life" (Asimov, 1990, pp. 275-276). ${ }^{31}$

En la obra se llega a plantear que nuestro propio universo quizá surgió de un modo parecido, bien fuere por una intervención de otros seres inteligentes, bien por el azar cuántico. De esta manera, Asimov extiende el ciclo destrucción-creación al origen mismo del universo. Una idea similar ya había sido explorada por él en su relato corto "The last question" (1956), donde una computadora, tras milenios de reflexión sobre si es posible revertir el inevitable final

31 [De hecho, al inducir las condiciones requeridas para el big bang, es posible que iniciemos una nueva clase de universo que eventualmente sea adecuado para la vida]. 
del universo, alcanza finalmente una respuesta. Ello se produce mucho tiempo después de que el ser humano haya desaparecido, pero aun así crea un nuevo universo.

\section{Élites}

Un segundo tema que aparece con frecuencia en la producción novelística de Asimov es el concepto de élite gobernante, con unos planteamientos que resultan muy próximos a los de La República de Platón, principalmente en lo que se refiere a la falta de escrúpulos para gobernar a las masas, aun en contra de sus propios deseos o intereses individuales: "de la mentira y el engaño es posible que hayan de usar muchas veces nuestros gobernantes por el bien de sus gobernados" (Platón, s. f.). En Foundation, son los oradores de la Segunda Fundación, y en The End of Eternity, los eternos, individuos que, amparados en unas capacidades intelectuales muy superiores a la media y armados con un conocimiento científico oculto al resto de la humanidad, rigen el destino de la raza.

Comenzando con los miembros de la Segunda Fundación, se trata de una suerte de élite intelectual quienes, reclutados en la infancia por poseer unas capacidades mentales excepcionales, están formados en las matemáticas de la psicohistoria. En la medida en que estos oradores pueden predecir la historia, también pueden alterarla, algo que no tienen reparo en hacer, obedeciendo a un plan cuyo fin último es el surgimiento de un nuevo imperio galáctico que, lógicamente, ellos gobernarían desde las sombras. Su actitud — soberbia y desdeñosa-, hacia el resto de seres humanos, queda perfectamente reflejada en los pensamientos de su líder, el Primer Orador: "[f]or twenty-five years, he, and his administration, had been trying to force a Galaxy of stubborn and stupid human beings back to the path" (Asimov, 2004g, p. 117)..$^{32}$

En relación con los miembros de La Eternidad, también son reclutados en su infancia y, al igual que sucede con los oradores, sometidos a un arduo proceso de instrucción que solo los más capaces

32 [Durante veinticinco años, él y su administración habían intentado obligar a toda la Galaxia, llena de tercos y estúpidos seres humanos, a reemprender aquel camino]. 
superan satisfactoriamente, quedando el resto relegados a tareas menores dentro de la organización. Como no podría ser de otra manera, este entrenamiento se articula en torno a la formación científica en diversas áreas relacionadas con los viajes en el tiempo. Ambas élites basan su poder en el intelecto, y no en la fuerza física; poseen un conocimiento científico de incalculable valor, que, sin embargo, esconden a aquellos que no forman parte de su organización; y se consideran custodios de la raza humana, con derecho a decidir su destino, convencidos de que su guía es una mejor alternativa que el libre albedrío.

La crítica que realiza Asimov a estas élites se articula en torno a tres ejes diferentes. En primer lugar, la falta de ética de sus miembros, firmes creyentes en la máxima maquiavélica de que "el fin justifica los medios", 33 es puesta en entredicho. Los oradores de la Segunda Fundación, entre otras cuestionables acciones, permiten que un planeta entero sea bombardeado y arrasado como parte de un engaño a su enemigo, The Mule, costando millones de vidas. El líder de la organización, si bien se muestra consternado, justifica esta acción como el mal menor: "The destruction of Tazenda was unavoidable. The alternative would have been a much greater destruction generally throughout the Galaxy over a period of centuries. We did our best in our limited way" (Asimov, 2004g, p. 89). ${ }^{34}$

Este mismo argumento de inevitabilidad es utilizado, otra vez, cuando la Segunda Fundación decide sacrificar a cincuenta miembros de su organización como parte de un nuevo plan para engañar, en esta ocasión, a la Primera Fundación:

Fifty martyrs! They knew it meant death or permanent imprisonment and they could not even be oriented to prevent weakening [...] -Might they have been fewer? — asked the Student, doubtfully. The

33 Así como el convencimiento de que la mentira ha de ser considerada como una herramienta, perfectamente lícita, de organización social. Como señala Peiró Muñoz, "Pocos autores han planteado el uso de la mentira en relación a cómo se organiza -o debiera organizarse - una sociedad de forma tan abierta como lo hicieron Platón y Maquiavelo. No son, desde luego, los únicos, pero sí dos de los más representativos" (2015, p. 33).

34 [La destrucción de Tazenda era inevitable. La alternativa hubiera sido una destrucción mucho mayor en toda la Galaxia durante muchos siglos. Hemos hecho lo que podíamos con nuestros medios limitados]. 
First Speaker slowly shook his head, - It was the lower limit. Less could not possibly have carried conviction-(Asimov, 2004g, p. 273). ${ }^{35}$

En lo que se refiere a los eternos, encontramos ejemplos muy similares, como la conversación entre el protagonista, Harlan, y un sociólogo de La Eternidad:

- Now the M.N.C. recommended,- - said Harlan (he spoke coolly, evenly, pronouncing the Standard Intertemporal Language in precise syllables), - by your Section involves induction of an accident in space and the immediate death by fairly horrible means of a dozen or more men. —Unavoidable—, said Voy, shrugging (Asimov, 2000, p. 12). ${ }^{36}$

De hecho, el protagonista de la novela, al reflexionar sobre sus actividades, se muestra ciertamente dubitativo acerca de la conveniencia de los cambios en la realidad:

He had handled Reality. He had tampered with a mechanism during a quick few minutes taken out of the 223rd and, as a result, a young man did not reach a lecture on mechanics he had meant to attend. He never went in for solar engineering, consequently, and a perfectly simple device was delayed in its development a crucial ten years. A war in the 224th, amazingly enough, was moved out of Reality as a result. Wasn't that good? What if personalities were changed? The new personalities were as human as the old and as deserving of life. If some lives were shortened, more were lengthened and made happier. A great work of literature, a monument of Man's intellect and feeling, was never written in the new Reality, but several copies were preserved in Eternity's libraries, were they not? And new creative works had come into existence, had they not? Yet that night Harlan spent hours in a hot agony of wakefulness, and when he finally drowsed groggily, he did something he hadn't done in years. He dreamed of his mother (Asimov, 2000, p. 33). ${ }^{37}$

35 [iCincuenta mártires! Sabían que significaba la muerte o una prisión perpetua, y ni siquiera podían ser orientados para impedir el debilitamiento [...] — ¿No podrían haber sido menos? — preguntó el estudiante, dubitativo. El Primer Orador negó lentamente con la cabeza. - Era el límite más bajo. Menos no hubieran podido aportar la convicción necesaria].

36 [-Ahora el C.M.N. —dijo Harlan, hablando en tono frío, silabeando el Idioma Pantemporal Normalizado con meticulosidad - recomendado por su sección, implica la inducción de un accidente espacial, y una muerte inmediata y bastante horrible para una docena o más de personas. -Es inevitable —dijo Voy, encogiéndose de hombros].

37 [Había manipulado la Realidad. Había descompuesto aquel mecanismo durante unos rápidos minutos sustraídos al siglo 223 , y, debido a ello, un joven no llegó a tiempo 
En segundo lugar, Asimov nos muestra que, pese a cubrirse con el manto del bien común, en muchos casos estas élites se mueven por intereses egoístas y mezquinos. El protagonista de The End of Eternity, al final de la novela, comprende este hecho, lo que en última instancia le impulsa a decidirse a destruir La Eternidad:

He thought of Computers, struggling against themselves, of Finge intriguing against Twissell and Twissell spying on Finge. He thought of Sennor, fighting his bald head by fighting all the Eternals [...]. And he saw Eternity with great clarity as a sink of deepening psychoses, a writhing pit of abnormal motivation, a mass of desperate lives torn brutally out of context (Asimov, 2000, pp. 188-189). ${ }^{38}$

En esta misma línea, en Foundation's Edge, el orador Stor Gendibal, hacia el final de la novela, muestra bien a las claras sus ambiciones:

Shandess will retire and I will be First Speaker. Then will come my chance to make us an active factor in revolutionizing the Galaxy [...]. I will be the most important and the most powerful scholar of them all (Asimov, 2004e, p. 436). ${ }^{39}$

Su opinión sobre el resto de oradores también nos deja ver que esta actitud no es exclusivamente suya:

para asistir a una conferencia sobre mecánica. Como resultado, nunca se interesó por la ingeniería solar, y un sencillo invento retrasó su aparición en diez años críticos. La consecuencia fue que una guerra en el siglo 224, sorprendentemente, desapareció de la Realidad. ¿No era aquello un bien? ¿Qué importaba que se modificasen las personalidades? Las nuevas personalidades eran tan humanas como las anteriores y tan merecedoras de vivir. Si la vida de algunas personas resultaba acortada, otras, en cambio, serían más largas y felices. Una gran obra de literatura, un monumento a la inteligencia y sensibilidad del Hombre, nunca se escribió en la nueva Realidad, pero varias copias de ella se conservaban en las bibliotecas de la Eternidad, ¿no era cierto? En cambio, fueron creadas otras nuevas obras. A pesar de todo ello, aquella noche Harlan pasó muchas horas atormentado por el insomnio, y cuando finalmente consiguió dormirse, ocurrió algo que no había sucedido en muchos años. Soñó con su madre].

38 [Pensó en los Programadores luchando entre sí, en Finge intrigando contra Twissell y Twissell ordenando que se espiaran las acciones de Finge. Pensó en Sennor, luchando contra su cuerpo sin pelo y al mismo tiempo contra todos los Eternos. [...] Y ahora contemplaba a la Eternidad como sumidero de morbosas psicosis, un pozo maligno de motivos anormales, una masa de vidas desesperadas arrancadas brutalmente de su curso normal].

39 [Shandess se retirará y yo seré primer orador. Entonces tendré la oportunidad de hacer que seamos un factor activo en la revolución de la Galaxia [...]. Seré el sabio más importante y poderoso de todos]. 
[...] all minds at the Speaker's Table were open [...] and Gendibal knew that what they felt was envy. So did they; just as Gendibal knew his own attitude was defensive, overcompensating ambition. And so did they (Asimov, 2004e, p. 129). ${ }^{40}$

Por último, y aquí encontramos una interesante crítica a la corriente abolicionista del transhumanismo, ${ }^{41}$ Asimov cuestiona de modo feroz que la humanidad deba ser protegida y guiada. En ambos casos, aquel explora las consecuencias de esta dictadura benevolente, cuyos efectos secundarios se revelan como muy perjudiciales, pues en las dos situaciones se muestra como un callejón sin salida que, al coartar la libertad, ciertamente disminuye el sufrimiento de la humanidad, pero al precio de volverla estática y abocarla a su desaparición. En Foundation's Edge, durante la discusión sobre la mejor forma de organización del Segundo Imperio, uno de los personajes sintetiza de manera perfecta este punto:

The Second Galactic Empire-worked out after the fashion of Trantor [la Segunda Fundación] — will be a paternalistic Empire, established by calculation, maintained by calculation, and in perpetual living death by calculation. It will be a dead end (Asimov, 2004e, p. 424).42

Del mismo modo, hacia el final de The End of Eternity, el protagonista es aleccionado sobre las razones por las que La Eternidad ha de ser destruida: "Can you understand that in averting the pitfalls and miseries that beset man, Eternity prevents men from finding their own bitter and better solutions, the real solutions that come from conquering difficulty, not avoiding it?" (Asimov, 2000, p. 184).43 De hecho, en esta novela se plantea que solo la desaparición de La

40 [todas las mentes de la Mesa de Oradores estaban abiertas [...] y Gendibal sabía que lo que sentían era envidia. Ellos también lo sabían, del mismo modo que Gendibal sabía que su propia actitud era defensiva, sobrecompensando su ambición. Y ellos tampoco lo ignoraban].

41 La corriente de pensamiento abolicionista propugna por el uso de la tecnología para eliminar el sufrimiento humano, tanto en su vertiente física como psicológica.

42 [El Segundo Imperio Galáctico — desarrollado a la manera de Trántor- será un Imperio paternalista, establecido por el cálculo, mantenido por el cálculo, y en perpetua muerte en vida por el cálculo. Será un callejón sin salida].

43 [iNo comprendes que al impedir las miserias y fracasos que torturan al Hombre, la Eternidad no le deja encontrar sus propias soluciones, difíciles pero provechosas, las soluciones verdaderas que se obtienen al vencer las dificultades, no al evitarlas?]. 
Eternidad evitará la extinción de la raza humana, que, recluida en un único planeta,

[...] knew Earth for what it was: a prison surrounded by an infinity of freedom... And mankind died out [...]. [T] here was a loss of purpose, a sense of futility, a feeling of hopelessness that could not be overcome. Eventually there was one last decline of the birth rate and finally, extinction. Your Eternity did that (Asimov, 2000, p. 183).44

Este es un tema que Asimov, de hecho, explora también en sus relatos y novelas de robots. En estas novelas, que forman parte de la metasaga Foundation, describe la pugna entre los humanos que permanecen en la Tierra y aquellos que optaron por colonizar nuevos planetas. Estos segundos deciden llevar consigo a sus asistentes robot, custodios permanentes de su bienestar y encargados de asegurarles una vida larga y carente de dificultades. Es esta carencia de desafíos la que lleva a que la batalla se incline del lado de los terrestres, que finalmente son quienes encabezan la expansión de la humanidad, mientras que los espaciales languidecen hasta desaparecer en sus respectivos planetas. Con la lección aprendida, se establece un fuerte tabú en torno al uso de robots, y "[t]he new worlds were founded fresh and they did not even want to remember their bitter humiliation as children under robot nursemaids" (Asimov, 2004e, pp. 384-385). ${ }^{45}$

Es necesario mencionar que la idea del progreso mediante la superación de desafíos muy probablemente se deba a la influencia del historiador Arnold J. Toynbee, ya mencionado antes, quien opinaba que las civilizaciones crecen y progresan en la medida en que se enfrentan a retos que son capaces de solventar de manera satisfactoria. En su opinión, ante la ausencia de desafíos, la civilización decae, precisamente lo que se describe en los tres ejemplos mencionados en este apartado (Toynbee, 1934-1961).

Para terminar con esta reflexión, en The Gods Themselves (1990) encontramos el mismo concepto de élite maquiavélica, que en esta

44 [[...] comprendió qué era la Tierra: una prisión en medio de una libertad infinita... Y la humanidad languideció hasta morir [...] Había una sensación de futilidad, una desesperanza que no pudo ser superada. Hubo un último descenso de la tasa de natalidad y, finalmente, la humanidad desapareció. Tu Eternidad hizo esto].

45 [Los nuevos mundos fueron fundados con otros criterios y los fundadores no quisieron ni recordar su amarga humillación de niños sometidos a niñeras-robots]. 
novela Asimov extiende a la civilización alienígena descrita en su segunda parte. Los "Seres duros", élite gobernante del planeta, son quienes desarrollan la tecnología de intercambio de materia, conscientes en todo momento de que esto supondrá la destrucción de nuestro sistema solar. De nuevo, se trata de individuos mucho más inteligentes que la población de "Seres blandos", y otra vez no dudan en recurrir al engaño para lograr sus objetivos. Respecto a la primera afirmación, Odeen, uno de los protagonistas, señala que "Hard Ones are so intelligent that a Soft One is nothing in comparison" (Asimov, 1990, p. 113). ${ }^{46}$ En relación con la segunda, esta élite, además de mostrarse completamente insensible respecto a la destrucción de nuestro sistema solar, mantiene en el permanente engaño a los "Seres blandos", ocultándoles lo que son en realidad: la etapa inmadura de su raza, que, una vez cumplida su misión reproductora, se transformará en "Seres duros",

Así pues, las élites son relativamente frecuentes en la producción novelística de Asimov, y siempre con unas características muy similares. Dada su concepción racionalista del mundo, su poder provendría del conocimiento y la ciencia, no de la fuerza física o la potencia militar. De hecho, otro rasgo distintivo de estas élites es que, más allá de sus conocimientos científicos, se hallan inermes ante cualquier amenaza violenta, por lo que de forma invariable han de recurrir al intelecto para superar las amenazas que se les plantean. Del mismo modo, llama la atención la ausencia de ética que las caracteriza, dispuestas a cualquier cosa con tal de alcanzar sus objetivos, sin preocuparse excesivamente, o incluso en absoluto, de los daños colaterales de sus acciones. "El fin justifica los medios", parecen decirnos, y esta actitud es la causa de su eliminación: no parece que Asimov considerase legítimo este tipo de comportamientos, ${ }^{47}$ y por ello las élites no suelen lograr sus metas, pese a tener, en apariencia, todo a su favor. En una entrevista con James Gunn (2005a, pp. 47-48), el propio Asimov señalaba que, sin tener claro cómo podría haberlo hecho, de continuar

46 [Los Seres Duros son tan inteligentes que un Ser Blando no es nada en comparación con ellos].

47 Opinión que ya había adelantado, entre otros, Patrouch (1974, p. 95). En esta misma línea, White (2005, p. 16) destaca el carácter humanitario de Asimov, algo que sin duda es incompatible con aceptar los comportamientos descritos anteriormente. 
con la metasaga Foundation, habría sido la primera Fundación la que habría resultado triunfadora sobre la Segunda, debido a su carácter democrático. ${ }^{48}$

No obstante, llegados a este punto, es necesario indicar que algunos autores, como Miller (2004), no comparten esta opinión. Profundizando en los planteamientos de Warrick (1977), Miller sostiene, al analizar específicamente la Eternidad y la Segunda Fundación que ya hemos mencionado, ${ }^{49}$ que sus novelas no son sino intentos iterativos por encontrar soluciones a los problemas que plantea la ética utilitarista (2004, p. 189)..$^{50}$ Según esta perspectiva, Asimov no rechaza La Eternidad porque esté en contra de su filosofía utilitarista, como hemos argumentado en los párrafos anteriores, sino que la rechaza, debido a que los eternos son malos utilitaristas, pues, de hecho, fracasan en su objetivo de lograr el mayor bien posible para el mayor número de individuos posible.

En el caso de la Segunda Fundación, Miller (2004, p. 199) sí considera que Asimov se sentía incómodo con su paternalismo de corte dictatorial, siendo esta la razón que, desde su punto de vista, lo lleva a buscar otras formas alternativas de organización social. Es decir, de las tres razones que se han expuesto en este trabajo para explicar el rechazo de Asimov a las élites gobernantes, Miller únicamente reconoce la tercera, y sostiene que este escritor no se sentía cómodo con La Eternidad ni con la Segunda Fundación, por su incapacidad para implementar con eficacia un programa utilitarista, más que por consideraciones de otra naturaleza. Por el contrario, nuestro punto de vista, que hemos buscado demostrar en este trabajo, es que su rechazo se debe a consideraciones de tipo ético, más que práctico.

48 Sabemos, por las secuelas que escribió posteriormente, que si bien no fue la Primera Fundación la triunfadora, tampoco lo fue la Segunda, siendo ambas superadas por "Galaxia", una forma de organización social que se sugiere en las últimas entregas de la metasaga Foundation, y que extrapola la hipótesis Gaia a nivel interplanetario: un superorganismo integrado por todos los seres vivos de la galaxia.

49 Así como "Galaxia".

50 Esta teoría ética sostiene que, moralmente, la mejor acción es la que genera la mayor utilidad para el mayor número de individuos, es decir, la que maximiza la utilidad a nivel global. 


\section{Conclusiones}

Como hemos visto, gran parte de la producción novelística de Asimov está construida con base en patrones que se repiten, condicionando desde la trama hasta la propia estructura. Es algo que no solo observamos en sus obras de ciencia ficción, sino que se extiende a otros géneros que también abordó.

En los relatos de misterio recogidos en Tales of the Black Widowers (1974) y More Tales of the Black Widowers (1976), la estructura es siempre idéntica, con un invitado que presenta un misterio ante los miembros del club de los viudos negros. Tras plantear distintas especulaciones y debatir posibles explicaciones, invariablemente es el mayordomo quien resuelve el enigma. Los mismo sucede con los relatos de fantasía satírica de Azazel, publicados entre 1982 y 1994 en Isaac Asimov's Science Fiction Magazine, ${ }^{51}$ en los que el protagonista pide un deseo a una especie de demonio alienígena en miniatura, deseo que, tras ser concedido, siempre produce efectos secundarios indeseables, que generan justo lo contrario a lo que se pretendía conseguir. En ambos casos, la estructura de los relatos es idéntica, siguiendo el mismo patrón que se repite una y otra vez, de manera que el lector ya conoce de antemano qué es lo que va a suceder.

En este trabajo hemos analizado dos esquemas que resultan especialmente interesantes en su obra: el ciclo creación-destrucción en lo que se refiere a la historia, y la existencia de una élite gobernante de tintes platónicos/maquiavélicos. Ambos patrones aparecen de manera recurrente en distintas novelas y relatos, siempre con características muy similares. Consciente o inconscientemente, Asimov tendía a incorporar en sus historias estos elementos, muy probablemente como consecuencia de su fascinación por los patrones que mencionábamos al principio de este trabajo. En sus obras, los ciclos históricos aparecen en distintas escalas de tiempo, que abarcan desde los pocos años, como sucede en la primera novela de la metasaga Foundation, hasta los miles de años, como observamos al analizar la

51 Varios de estos relatos fueron compilados posteriormente en distintos libros. El que recoge un mayor número de estas historias es el titulado precisamente Azazel (Asimov, 1988). 
saga completa, o incluso los muchos millones de años, cuando trata la propia existencia del universo en "The last question". Pero sucede siempre con ese carácter recurrente que Asimov interpretaba como inherente a la naturaleza de la historia.

Respecto al segundo patrón, las élites gobernantes, Asimov las visualizaba conformadas por individuos hiperracionales, custodios de la ciencia y el conocimiento, que, cargando sobre sus hombros la responsabilidad del bien común, en algún momento se extraviaron. No hay dudas sobre la convicción de estas clases gobernantes respecto al papel que han de desempeñar, pues todos sus miembros, sin excepción, se ven como salvadores de la humanidad, responsables de dirigir al ser humano por el camino correcto, mientras velan por su protección frente a las amenazas del destino. Probablemente constituía una carga demasiado pesada, que les lleva a alejarse del sendero, lo que en última instancia ocasiona su desaparición. ${ }^{52}$

El mensaje es doble: el conocimiento, sin una ética que lo dirija, es el camino a la perdición, y el libre albedrío resulta clave para el progreso de la humanidad, pues, sin desafíos que nos pongan a prueba, el ser humano languidece inexorablemente.

\section{Agradecimientos}

Quisiera agradecer a los evaluadores y al corrector del artículo sus comentarios y observaciones, que han contribuido a mejorar sustancialmente este trabajo $\mathbf{I}$

52 Realmente, Asimov mostraba una cierta preocupación a este respecto, pues consideraba que si bien un Gobierno Mundial a nivel planetario podría ser deseable para afrontar desafíos de carácter global, como los problemas medioambientales, se corría el riesgo de su transformación en una dictadura universal y opresiva (Asimov, 1986, p. 91). 


\section{Referencias}

Arroyo Barrigüete, J. L. (2018). Hibridación genérica en ciencia ficción: análisis architextual de la trilogía Foundation de Asimov. Anuario de Estudios Filológicos, 41, 5-25.

Asimov, I. (1941). Nightfall. Astounding Science Fiction. Recuperado de http://mysite. du.edu/ treddell/3780/Asimov_Nightfall.pdf

Asimov, I. (1950a). I, Robot. Nueva York: Gnome Press.

Asimov, I. (1950b). Pebble in the Sky. Nueva York: Doubleday.

Asimov, I. (1951). The Stars, Like Dust. Nueva York: Doubleday.

Asimov, I. (1952). The Currents of Space. Nueva York: Doubleday.

Asimov, I. (1954). The Caves of Steel. Nueva York: Doubleday.

Asimov, I. (1956). The last question. Science Fiction Quarterly, Nov. 1956. Recuperado de https://www.physics.princeton.edu/ph115/LQ.pdf

Asimov, I. (1957). The Naked Sun. Nueva York: Doubleday.

Asimov, I. (1974). Tales of the Black Widowers. Nueva York: Doubleday.

Asimov, I. (1976). More Tales of the Black Widowers. Nueva York: Doubleday.

Asimov, I. (1983). The Robots of Dawn. Nueva York: Doubleday.

Asimov, I. (1985). Robots and Empire. Nueva York: Doubleday.

Asimov, I. (1986). Sobre la ciencia ficción. Barcelona: Edhasa.

Asimov, I. (1988). Azazel. Nueva York: Doubleday.

Asimov, I. (1990). The Gods Themselves. New York: Bantam Books, Random House Publishing Group. Edición original: 1972.

Asimov, I. (2000). The End of Eternity. Londres: HarperCollins. Edición original: 1955.

Asimov, I. (2004a). Forward the Foundation. Nueva York: Bantam. Edición original: 1993.

Asimov, I. (2004b). Foundation. Nueva York: Bantam. Edición original: 1951.

Asimov, I. (2004c). Foundation and Earth. Nueva York: Bantam. Edición original: 1986.

Asimov, I. (2004d). Foundation and Empire. Nueva York: Bantam. Edición original: 1952.

Asimov, I. (2004e). Foundation's Edge. Nueva York: Bantam. Edición original: 1982.

Asimov, I. (2004f). Prelude to Foundation. Nueva York: Bantam. Edición original: 1988.

Asimov, I. (2004g). Second Foundation. Nueva York: Bantam. Edición original: 1953.

Asimov, I., y Silverberg, R. (1990). Nightfall. Nueva York: Doubleday.

Bajtín, M. (1989) Teoría y estética de la novela. Trad. H. Kriukova y V. Cazcarra. Madrid: Tauros. Edición original: 1924.

Berger, A. I. (1979). Nuclear energy: Science fiction's metaphor of power. Science Fiction Studies, 6(2), 121-128. 
Booker, M. K. (2001). Monsters, Mushroom Clouds, and the Cold War: American Science Fiction and the Roots of Postmodernism, 1946-1964. Westport: Greenwood.

Bowler, P. J. (2017). A History of the Future: Prophets of Progress from H.G. Wells to Isaac Asimov. Cambridge: Cambridge University Press, Edición de Kindle.

Chaves, J. R. (2016). Monstruos fantásticos en la literatura costarricense. Revista de Filología y Lingüística de la Universidad de Costa Rica, 42(Especial), 77-89. DOI: 10.15517/rfl. $\mathrm{v} 42 \mathrm{i} 0.26466$

Clute, J. (2007). Isaac Asimov. En D. Seed (Ed.), A Companion to Science Fiction (pp. 364374). Oxford: Blackwell.

Correia Félix, J. F. (2014). Symbolic Utopias: Herbert, Asimov and Dick (Tesis doctoral). Universidad de Sussex, Falmer, Reino Unido. Recuperado de http://sro.sussex.ac.uk/id/ eprint $/ 53989$

Csicsery-Ronay Jr., I. (2008). The Seven Beauties of Science Fiction. Middletown: Wesleyan University Press.

Fanzo, D. A. (1965). An Inquiry into the Literature of Science Fiction: Its Development, Maturation, and Significance as a Literary Genre (Tesis de máster). Universidad Texas Tech, Texas, Estados Unidos.

Fitting, P. (1979). The modern Anglo-American sF novel: Utopian longing and capitalist cooptation. Science Fiction Studies, 6(1), 59-76. Recuperado de https://www.depauw. edu/sfs/backissues/17/fitting17.htm.

Genette, G. (1989). Palimpsestos: la literatura en segundo grado. Traducción de C. Fernández Prieto. Madrid: Taurus.

Goble, N. (1972). Asimov Analyzed. Maryland: The Mirage Press.

Gunn, J. (2005a). An interview with Isaac Asimov. En C. Freedman (Ed.), Conversations with Isaac Asimov (pp. 34-55). Jackson: The University Press of Mississippi.

Gunn, J. (2005b). The Foundations of Science Fiction. Maryland: Scarecrow.

Gunn, J. (2018). Alternate Worlds. The Illustrated History of Science Fiction. Jefferson: McFarland \& Company.

Hark, I. R. (1979). Unity in the Composite Novel: Triadic Patterning in the Asimov's The Gods Themselves. Science Fiction Studies, 6(3), 281-286. Recuperado de https://www. depauw.edu/sfs/backissues/19/hark19art.htm.

Hassler, D. M. (1988). Some Asimov resonances from the enlightenment. Science Fiction Studies, 15(1), 36-47.

Heinlein, R. A. (1959). All You Zombies. The Magazine of Fantasy $\mathcal{E}$ Science Fiction. Recuperado de http://www.uky.edu/ mwa229/AllYouZombies.pdf.

Ingersoll, E. G. (2005). A conversation with Isaac Asimov. En C. Freedman (Ed.), Conversations with Isaac Asimov (pp. 21-33). Jackson: The University Press of Mississippi. 
Irwin, R. (1997). Toynbee and Ibn Khaldun. Middle Eastern Studies, 33(3), 461-479.

James, E. (2015). Lois McMaster Bujold. Baltimore: University of Illinois Press.

Käkelä, J. (2014). Managing and manipulating history: Perpetual urgency in Asimov and Heinlein. Fafnir, 1(2), 7-22.

Käkelä, J. (2016). The Cowboy Politics of an Enlightened Future: History, Expansionism, and Guardianship in Isaac Asimov's Science Fiction (Tesis doctoral). Universidad de Helsinki, Helsinki, Finlandia.

Martínez, R. (2012). La ciencia ficción de Isaac Asimov. España: Sportula.

Miller, J. J. (2004). The greatest good for humanity: Isaac Asimov's future history and utilitarian calculation problems. Science-Fiction Studies, 31(2), 189-206.

Milner, A., y Savage, R. (2008). Pulped dreams: Utopia and American pulp science fiction. Science Fiction Studies, 35(1), 31-47.

Novell Monroy, N. (2008). Literatura y cine de ciencia ficción. Perspectivas teóricas (Tesis doctoral). Universidad Autónoma de Barcelona, Barcelona, España.

Palumbo, D. E. (1995). Psychohistory and chaos theory: The 'Foundation Trilogy' and the fractal structure of Asimov's Robot/Empire/Foundation Metaseries. Journal of the Fantastic in the Arts, 7(1), 23-50.

Palumbo, D. E. (2016). An Asimov Companion: Characters, Places and Terms in the Robot/ Empire/Foundation Metaseries. Jefferson: McFarland \& Co Inc. Edición de Kindle.

Patrouch, J. F. (1974). The Science Fiction of Isaac Asimov. Nueva York: Doubleday.

Peiró Muñoz, F.A. (2015). Platón y Maquiavelo. En torno a la posibilidad de una "noble mentira" en política. Actas I Congreso internacional de la Red española de Filosofía, vol. 6, 33-45.

Pez, J. (s. f.). The History of the Positronic Robot and Foundation Stories. Part 2: 1941-1944. Recuperado de http://www.asimovonline.com/oldsite/Robot_Foundation_history_2. html.

Platón (s. f.). La República (Spanish Edition). España: Debrus Producciones, Edición de Kindle.

Polibio. (2008). Historias. Libro VI. Trad. Antonio Sáncho Royo. Madrid: Consejo Superior de Investigaciones Científicas, Tirant lo Blanch.

Roberts, A. (2017). From gothic to science fiction. En J. Holmes y S. Ruston (Eds.), The Routledge Research Companion to Nineteenth-Century British Literature and Science (pp. 87100). Abingdon: Routledge. Edición de Kindle.

Sawyer, A. (2009). Future history. En M. Bould, A. Butler, A. Roberts y S. Vint (Eds.), The Routledge Companion to Science Fiction (pp. 489-493). Nueva York: Routledge. 
Seed, D. (2011). Science Fiction: A very Short Introduction. Nueva York: Oxford University Press.

Shippey, T. (2016). Hard Reading: Learning from Science Fiction. Liverpool: Liverpool University Press.

Shippey, T., Stableford, B. M., Feeley, G. y Langford, D. (2015). History in SF. En J. Clute, D. Langford, P. Nicholls y G. Sleight (Eds.), The Encyclopedia of Science Fiction. Londres: Gollancz. Recuperado de http://www.sf-encyclopedia.com/entry/history_in_sf.

Stableford, B. (1995). Frankenstein and the origins of science fiction. En D. Seed (Ed.), Anticipations: Essays on Early Science Fiction and Its Precursors (pp. 46-57). Syracuse: Syracuse University Press.

Stone, P. (2005). The Plowboy interview: Isaac Asimov - Science, technology... and space. En C. Freedman (Ed.), Conversations with Isaac Asimov (pp. 56-73). Jackson: The University Press of Mississippi.

Sutherland, J. A. (2014). American science fiction since 1960. En P. Parrinder (Ed.), Science Fiction: A Critical Guide (pp. 162-186). Nueva York: Routledge.

Toynbee, A. J. (1934-1961). A Study of History (Vol. I a XII). Oxford: Oxford University Press.

Warrick, P. S. (1977). Ethical evolving artificial intelligence. En J. D. Olander y M. H. Greeberg (Eds.). Isaac Asimov (pp. 174-200). Nueva York: Taplinger Publishing Company.

Webster, J. K. (2015). Science Fiction as a Prism for Understanding Geopolitics (Tesis de Master). Air University (Air Command and Staff College), Alabama, Estados Unidos.

Weldes, J. (2001). Globalisation is science fiction. Journal of International Studies, 30(3), 647667. DOI: $10.1177 / 03058298010300030201$.

White, M. (2005). Isaac Asimov. A Life of the Grand Master of Science Fiction. Nueva York: Carroll \& Graf Publishers.

Wolfe, G. K. (1986). Critical Terms for Science Fiction and Fantasy. A Glossary and Guide to Scholarship. Westport: Greenwood Press. 